ARTICLE

Received 19 May 2014 | Accepted 9 Oct 2014 | Published 25 Nov $2014 \quad$ DOl: 10.1038/ncomms6540

\title{
Diacylglycerol mediates regulation of TASK potassium channels by $\mathrm{Gq}$-coupled receptors
}

Bettina U. Wilke ${ }^{1}$, Moritz Lindner ${ }^{1} \dagger$, Lea Greifenberg ${ }^{1}$, Alexandra Albus ${ }^{1}$, Yannick Kronimus ${ }^{1}$, Moritz Bünemann², Michael G. Leitner ${ }^{1} \&$ Dominik Oliver ${ }^{1}$

The two-pore domain potassium (K2P) channels TASK-1 (KCNK3) and TASK-3 (KCNK9) are important determinants of background $\mathrm{K}^{+}$conductance and membrane potential. TASK-1/3 activity is regulated by hormones and transmitters that act through $\mathrm{G}$ protein-coupled receptors (GPCR) signalling via $G$ proteins of the $G \alpha_{q / 11}$ subclass. How the receptors inhibit channel activity has remained unclear. Here, we show that TASK-1 and -3 channels are gated by diacylglycerol (DAG). Receptor-initiated inhibition of TASK required the activity of phospholipase $\mathrm{C}$, but neither depletion of the PLC substrate $\mathrm{PI}(4,5) \mathrm{P}_{2}$ nor release of the downstream messengers $\mathrm{IP}_{3}$ and $\mathrm{Ca}^{2}{ }^{2}$. Attenuation of cellular DAG transients by DAG kinase or lipase suppressed receptor-dependent inhibition, showing that the increase in cellular DAG-but not in downstream lipid metabolites-mediates channel inhibition. The findings identify DAG as the signal regulating TASK channels downstream of GPCRs and define a novel role for DAG that directly links cellular DAG dynamics to excitability.

\footnotetext{
${ }^{1}$ Institute of Physiology and Pathophysiology, Department of Neurophysiology, Philipps University, Deutschhausstr. 1-2, 35037 Marburg, Germany. ${ }^{2}$ Department of Pharmacology and Clinical Pharmacy, Philipps University, 35032 Marburg, Germany. †Present address: Department of Ophthalmology, University of Bonn, Bonn, Germany. Correspondence and requests for materials should be addressed to D.O. (email: oliverd@staff.uni-marburg.de).
} 
D iacylglycerol (DAG) is one of the best-studied second messengers of eukaryotic cells ${ }^{1}$. It is released by stimulus-induced activation of phospholipase C (PLC), which cleaves the minor plasma membrane phospholipid phosphatidylinositol-4,5-bisphosphate $\left(\mathrm{PI}(4,5) \mathrm{P}_{2}\right)$ to produce DAG and inositol-1,4,5-trisphosphate $\left(\mathrm{IP}_{3}\right)$. The PLC $\beta$ isozymes are activated by $G$ protein-coupled receptors that signal through the $\mathrm{G}_{\mathrm{q} / 11}$ subclass of $\mathrm{G} \alpha$ proteins (GqPCRs). PLC $\beta$ activation occurs by direct interaction with the $G \alpha$ protein; $\beta / \gamma$ subunits and increased intracellular $\mathrm{Ca}^{2}+$ can also promote PLC $\beta$ activation ${ }^{2}$.

As a membrane-resident messenger, DAG operates not only by binding to target proteins, but also by recruiting DAG-binding proteins to the membrane $e^{1,3}$. Besides the prototypical DAG effector, protein kinase $\mathrm{C}$ (PKC), there is a structural and functional diversity of high-affinity DAG effector proteins ${ }^{1,4}$. However, common to all of these effectors is that the DAG interaction is mediated by cysteine-rich $\mathrm{C} 1$ domains, which mediate recruitment to the plasma membrane or activate the protein $^{5}$. Little is known about DAG effectors that do not use C1 domains. Some members of the canonical TRP cation channels (TRPC) can be activated by DAG. The binding site and the molecular mechanism of activation by DAG are unknown ${ }^{6}$. Beyond its function as a messenger in its own right, DAG is also a precursor in the stimulus-driven production of several signalling lipids, including phosphatidic acid (PA), the endocannabinoid 2 -arachidonoylglycerol (2-AG), free arachidonic acid and their downstream metabolites ${ }^{4}$.

The TWIK-related acid-sensitive $\mathrm{K}^{+}$channels, TASK-1 and TASK-3 (KCNK3 and KCNK9), constitute a subgroup of the twopore domain (K2P) potassium channel family ${ }^{7}$. These channels function as background or 'leak' channels that are open at resting potentials and thus provide a resting $\mathrm{K}^{+}$conductance that strongly contributes to setting the membrane potential. In excitable cells TASK channels control action potential threshold and spiking patterns ${ }^{8-10}$. TASK channels show a broad, yet selective expression pattern in many organs, including the central nervous system, peripheral chemoreceptors, vascular smooth muscle and cardiac muscle, and adrenal cortex ${ }^{7}$, consistent with the involvement in the regulation of a wide variety of physiological processes.

TASK-1 and -3 channels are extensively modulated by physiological stimuli. A defining property of TASK channels is closure on extracellular acidification near physiological $\mathrm{pH}^{11}$. Moreover, they are inhibited by hormones and transmitters that signal through GqPCRs ${ }^{12-15}$. The resulting cellular depolarization is thought to regulate processes such as motor control ${ }^{10,15,16}$ and aldosterone secretion ${ }^{17}$. The precise signal-transduction pathway downstream of GqPCRs that operates TASK inhibition has remained unresolved.

Here we show that DAG is both sufficient for TASK channel inhibition and necessary for downregulation by GqPCRs. In contrast, downstream intermediates of DAG had no or only a minor impact on channel activity. The results uncover a new and possibly widespread signalling role for the prototypic lipid messenger DAG. As TASK channels lack the archetypical C1 DAG-binding domain thought to mediate most cellular effects of $\mathrm{DAG}^{1,4}$, these channels may provide a new paradigm for $\mathrm{C} 1$-independent DAG effector proteins.

\section{Results}

PLC is required for TASK channel inhibition. Receptormediated inhibition of TASK has been proposed to result from either direct interaction with activated $\mathrm{G} \alpha_{\mathrm{q}}{ }^{18}$ or from the action of a messenger downstream of the activity of PLC $\beta^{12,19}$.
To differentiate between both candidate pathways, we first addressed the requirement for PLC $\beta$ in GqPCR-dependent channel inhibition.

We started by testing pharmacological inhibition of PLC $\beta$ by the widely used PLC inhibitor U73122 (ref. 20). Application of U73122 almost completely abrogated inhibition of TASK-3 channels in response to activation of the co-expressed Gq-coupled muscarinic M1 receptor (M1R) in Chinese hamster ovary (CHO) cells (Fig. 1a-c). The inactive analogue, U73343, had no effect on receptor-mediated channel inhibition. Because previously reported effects of U73122 on TASK regulation have been controversial ${ }^{12,18,19,21,22}$, we additionally controlled for the efficacy of U73122 under our experimental conditions. By using total internal reflection fluorescence microscopy (TIRF-M) measurements with the $\mathrm{PI}(4,5) \mathrm{P}_{2}$-binding translocation sensor PLC61-PH-GFP as a readout for PLC activity ${ }^{20,23,24}$, we confirmed that PLC $\beta$ was essentially disabled by preapplication of U73122 (Fig. 1d-f). In this assay the TIRF signal amplitude directly reports the degree of sensor binding to the membrane, and thus the concentration of its ligand $\mathrm{PI}(4,5) \mathrm{P}_{2}$. Therefore, fluorescence decrease indicates depletion of $\mathrm{PI}(4,5) \mathrm{P}_{2}$ and hence PLC activity. Pre-application of $5 \mu \mathrm{M}$ U73122, but not U73343, prevented translocation of PLC $\delta 1-\mathrm{PH}-\mathrm{GFP}$ from the membrane on activation of M1R. Equivalent results were obtained using a DAG sensor (PKC $\left.\gamma-C 1-G^{20,25}\right)$ for detection of PLC activity (not shown).

Previous work addressed the role of PLC by employing a double mutant of $\mathrm{G} \alpha_{\mathrm{q}}\left(\mathrm{G} \alpha_{\mathrm{q}}-\mathrm{AA}\right)$ thought to disable activation of PLC by active $\mathrm{G} \alpha_{\mathrm{q}}$. When expressed in cells lacking both $\mathrm{G} \alpha_{\mathrm{q}}$ and $\mathrm{G} \alpha_{11}\left(\mathrm{G} \alpha_{\mathrm{q} / 11}{ }^{-1-}\right.$ embryonic fibroblasts), $\mathrm{G} \alpha_{\mathrm{q}}-\mathrm{AA}$ reconstituted TASK channel inhibition, thus questioning the role of PLC in TASK channel regulation ${ }^{18}$. However, by using PKC $\gamma-C 1-G F P$ as a sensor of PLC activity we found that when expressed in $\mathrm{G} \alpha_{\mathrm{q} / 11}{ }^{-1-}$ cells, $\mathrm{G} \alpha_{\mathrm{q}}$-AA efficiently activated PLC on receptor stimulation with little difference to wild-type $\mathrm{G} \alpha_{\mathrm{q}}$ (Fig. 1g,h). Further introduction of additional three mutations reported to impair Gq/PLC-coupling $\left(\mathrm{G} \alpha_{\mathrm{q}}-5 \mathrm{~A}\right)^{26}$ only slightly affected PLC activation. Accordingly, both $\mathrm{G} \alpha_{\mathrm{q}}$ mutants restored receptormediated inhibition of TASK-3 in $\mathrm{G} \alpha_{\mathrm{q} / 11}{ }^{-/-}$fibroblasts (Fig. 1i,j). Recently, Zhang et al. ${ }^{27}$ developed a chimeric $\mathrm{G} \alpha_{\mathrm{q}}$ $\left(\mathrm{G} \alpha_{\text {qiq }}\right)$ in which the PLC interaction domain is replaced by the homologous region of $\mathrm{G} \alpha_{\mathrm{i}}$. This construct can still be activated by GqPCRs, but coupling to PLC is abrogated. When $\mathrm{G} \alpha_{\mathrm{qiq}}$ was co-expressed with the M1R receptor and the $\mathrm{PKC} \gamma-\mathrm{C} 1$ sensor domain in $\mathrm{G} \alpha_{\mathrm{q} / 11}$-deficient cells, activation of PLC was lacking (Fig. 1g,h), confirming elimination of functional coupling to PLC. As shown in Fig. 1i,j, G $\alpha_{\text {qiq }}$ was unable to restore M1R-mediated inhibition of TASK-3 in $\mathrm{G} \alpha_{\mathrm{q} / 11}-1-$ fibroblasts. We therefore conclude that activation of PLC by $\mathrm{G} \alpha_{\mathrm{q}}$ is an indispensable step for the receptor-mediated inhibition of TASK channels.

Intracellular $\mathrm{Ca}^{2+}$ at or above the physiological resting concentrations is an established requirement for GqPCRstimulated PLC $\beta$ activity ${ }^{20}$. We used this $\mathrm{Ca}^{2}+$ dependence as an additional criterion for assessing the involvement of PLC. When intracellular $\mathrm{Ca}^{2}+$ was clamped to values below physiological resting levels by inclusion of the $\mathrm{Ca}^{2+}$ chelator BAPTA $(20 \mathrm{mM})$ in the intracellular solution, PLC activity, as measured by translocation of the $\mathrm{PKC} \gamma-\mathrm{C} 1$ sensor, was indeed abrogated. Receptor-mediated inhibition of TASK-3 channels measured simultaneously from the same cells was also entirely abolished (Fig. 2a). Similarly, $20 \mathrm{mM}$ EGTA largely prevented inhibition of TASK, whereas $5 \mathrm{mM}$ BAPTA only partially occluded channel inhibition (Fig. 2b), which is similar to previous findings showing that buffering with $5 \mathrm{mM}$ BAPTA did not prevent TASK channel regulation ${ }^{12}$. As high buffer concentrations or extremely low intracellular $\mathrm{Ca}^{2}+$ levels might 
a

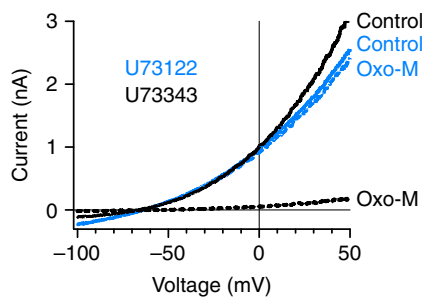

d

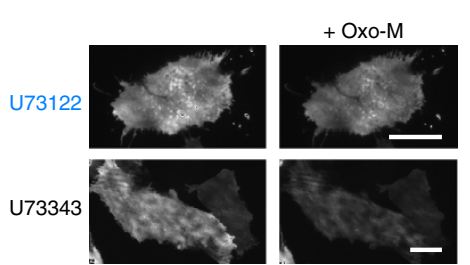

b

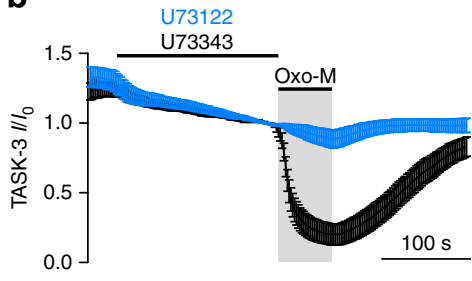

C

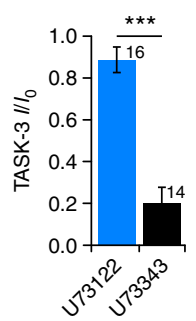

f

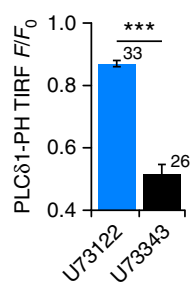

g

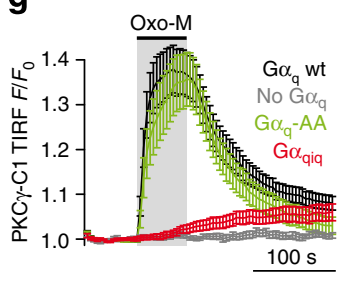

h

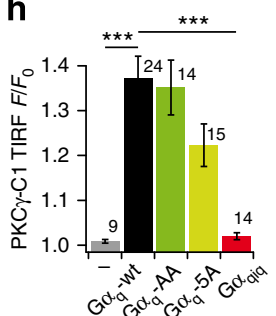

i

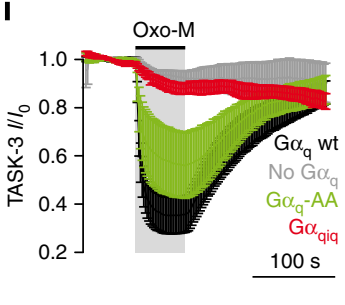

j

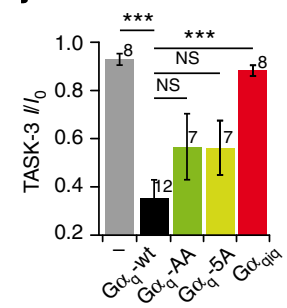

Figure 1 | Phospholipase C (PLC) is necessary for GqPCR-mediated TASK-3 inhibition. (a) PLC inhibitor U73122 disabled GqPCR-mediated inhibition of TASK-3. Representative $\mathrm{K}^{+}$currents from CHO cells co-transfected with hTASK-3 and M1R before (continuous) and after application of the muscarinic agonist, oxotremorine-M (Oxo-M, $10 \mu \mathrm{M}$ for all experiments; dashed trace). Recordings were made following application of either $5 \mu \mathrm{M}$ U73122 (blue) or the inactive analogue U73343 (black). (b) Average time course of TASK-3 currents at $+50 \mathrm{mV}$ from experiments as in (a). Currents were normalized to the amplitude immediately before application of Oxo-M. (c) Mean residual currents after application of Oxo-M. (d) Inhibition of PLC $\beta$ by U73122 was confirmed by the PI(4,5) $\mathrm{P}_{2}$ sensor PLC $81-\mathrm{PH}-\mathrm{GFP}$. Representative TIRF images of CHO cells co-transfected with PLC $81-\mathrm{PH}-\mathrm{GFP}$ and M1R before (left panels) and during application of $10 \mu \mathrm{M}$ Oxo-M (right panels). Scale bar, $20 \mu \mathrm{m}$. (e) Mean time course of single-cell TIRF intensity normalized to fluorescence before application of Oxo-M. (f) Mean normalized TIRF intensity at the end of the Oxo-M application. (g) M1R-induced activity of PLC was measured in $G \alpha_{q / 11}{ }^{-1}$ - fibroblasts co-transfected with DAG sensor PKC $\gamma$-C1-GFP, M1R, and either no $G \alpha_{q}$, wild-type $G \alpha_{q}$, mutant $G \alpha_{q}-A A$ or a $G \alpha_{q i q}$ chimera deficient in coupling to PLC. PLC activity was probed as the degree of membrane association of PKC $\gamma$-C1-GFP with TIRF microscopy. (h) Mean normalized increase in membrane association of PKC $\gamma$-C1-GFP at the end of the application of Oxo-M in the presence of G $\alpha_{q}$ or the mutant forms G $\alpha_{q}-A A$, $\mathrm{G} \alpha_{\mathrm{q}}-5 \mathrm{~A}$ or $\mathrm{G} \alpha_{\text {qiq }}$ (i,j) Differential inhibition of TASK-3 current by receptor activation in $\mathrm{G} \alpha_{\mathrm{q} / 11}{ }^{-/}$- fibroblasts when co-expressed with either wild type or mutant $\mathrm{G} \alpha_{\mathrm{q}}$. Error bars indicate s.e.m. ${ }^{\star \star \star} P<0.001$; ${ }^{\star \star} \mathrm{P}<0.01$; ${ }^{\star} P<0.05$. Numbers on bar graphs indicate the number of replicates (individual cells).

also affect other cellular processes, we further tested for the effects of this condition on signalling upstream of PLC activity. To this end we used a FRET assay to monitor receptor-induced interaction between $\mathrm{G} \alpha_{\mathrm{q}}$ and PLC $\beta$. As shown in Fig. $2 c$, the FRET signal between $\mathrm{G} \alpha_{\mathrm{q}}$-CFP and YFP-PLC $\beta 3$ rapidly increased on activation of co-expressed $\mathrm{M} 1 \mathrm{R}$ receptor, directly reporting the interaction. This increase in FRET was not disrupted by high intracellular BAPTA. Thus, upstream signalling including the function of $\mathrm{G} \alpha_{\mathrm{q}}$ remained functional, supporting the conclusion that the effect of BAPTA on TASK channel regulation resulted from inhibition of PLC.

Although it had been suggested that depletion of the PLC substrate, $\mathrm{PI}(4,5) \mathrm{P}_{2}$, may be the signal triggering channel deactivation ${ }^{12,28}$, we recently showed that depletion of $\mathrm{PI}(4,5) \mathrm{P}_{2}$ does not alter channel activity of TASK-1 and TASK-3 (ref. 29). Yet, the requirement for PLC implies an indirect role of its substrate $\mathrm{PI}(4,5) \mathrm{P}_{2}$, as a sufficient concentration of substrate must be present to allow for the production of any downstream signalling intermediate. We therefore probed the regulation of TASK channels during forced depletion of $\mathrm{PI}(4,5) \mathrm{P}_{2}$ and its precursor $\mathrm{PI}(4) \mathrm{P}$ by pseudojanin, an engineered chimeric phosphoinositide phosphatase that degrades $\mathrm{PI}(4,5) \mathrm{P}_{2}$ and $\mathrm{PI}(4) \mathrm{P}^{29,30}$. Pseudojanin was acutely activated by recruitment to the membrane via rapamycin-dependent heterodimerization (Fig. 2d $)^{29,31}$. Pseudojanin-mediated depletion of $\mathrm{PI}(4,5) \mathrm{P}_{2}$ and PI(4)P precluded PLC activity on subsequent activation of M1R as indicated by lack of DAG production (Fig. 2e) and abolished channel inhibition (Fig. 2f). As it was previously shown that transcriptional induction of a $\mathrm{PI}(4,5) \mathrm{P}_{2}$ 5-phosphatase did not affect regulation of TASK currents by $\mathrm{G}_{\mathrm{q}}$-coupled receptors ${ }^{18}$, we also tested for an effect of selective depletion of $\mathrm{PI}(4,5) \mathrm{P}_{2}$. To this end we used a pseudojanin construct in which the $\mathrm{PI}(4) \mathrm{P}$ 4-phosphatase SAC1 was inactivated by mutation (PJ-5Ptase). As shown in Fig. 2f, recruitment of PJ-5Ptase did not affect TASK regulation. However, monitoring the production of DAG under this condition revealed that the receptor-induced DAG transient was also unchanged (Fig. 2e). Thus, despite depletion of $\mathrm{PI}(4,5) \mathrm{P}_{2}$, PLC activity is not reduced to a measurable degree. Selective activation of a 5 -phosphatase may not be sufficient to lower $\mathrm{PI}(4,5) \mathrm{P}_{2}$ concentration enough to eliminate $\mathrm{PI}(4,5) \mathrm{P}_{2}$ 


\section{a}

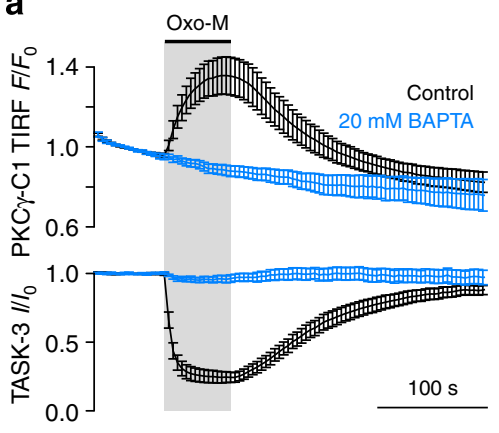

b

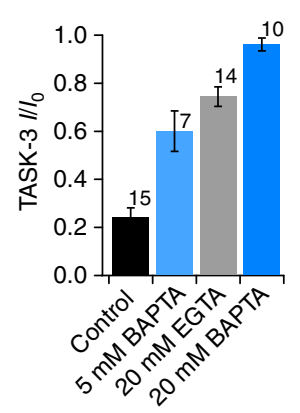

C

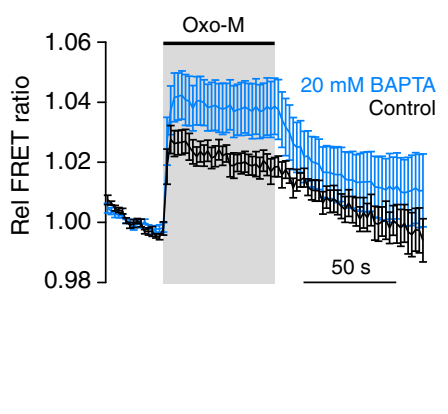

d

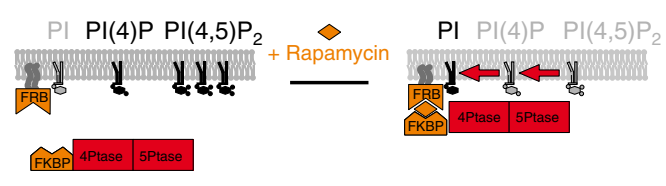

e

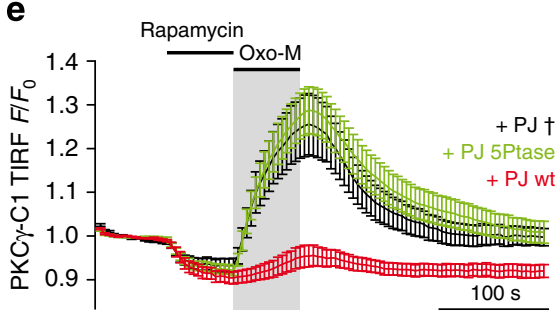

\section{g}

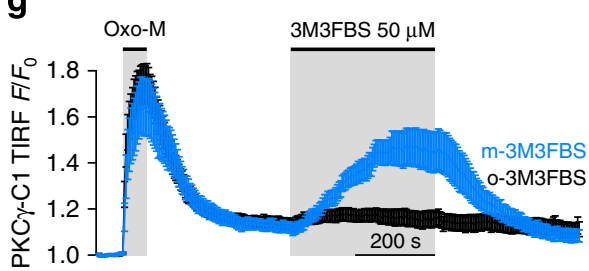

f

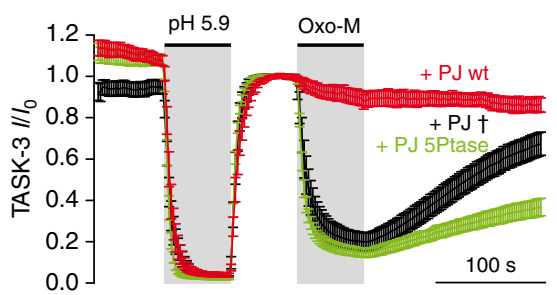

h

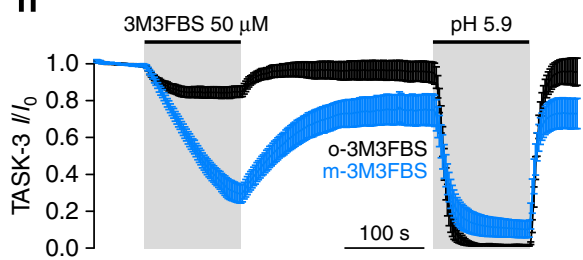

Figure 2 | Involvement of PLC in GqPCR-mediated TASK-3 inhibition. (a) Strong buffering of intracellular Ca ${ }^{2}+$ suppressed TASK-3 inhibition by M1R. CHO cells co-expressing TASK-3, DAG sensor PKC $\gamma$-C1-GFP and M1R were whole-cell patch-clamped with intracellular solutions buffered to either $0.1 \mu \mathrm{M}$ free $\mathrm{Ca}^{2+}$ (control) or without $\mathrm{Ca}^{2+}$ but $20 \mathrm{mM}$ of the $\mathrm{Ca}^{2+}$ chelator BAPTA. PKC $\gamma$-C1-GFP translocation was monitored simultaneously by TIRF microscopy. (b) Mean residual currents after application of $10 \mu \mathrm{M}$ Oxo-M with either $0.1 \mu \mathrm{M}$ intracellular $\mathrm{Ca}^{2}+$ (control) or the $\mathrm{Ca}^{2+}$ chelators indicated. (c) Dynamic FRET measurements between $\mathrm{G} \alpha_{\mathrm{q}}$-CFP and YFP-PLC $\beta 3$ were done with whole-cell patch-clamped cells after 4 min of equilibration with solutions containing either $0.1 \mu \mathrm{M}$ free $\mathrm{Ca}^{2}+($ control, $n=9)$ or $20 \mathrm{mM}$ BAPTA $(n=9)$. Rapid increase in FRET following activation of co-expressed M1R indicates comparable association of $G \alpha_{q}$ and PLC 33 in both conditions. (d) Principle of induced depletion of PLC substrate. Application of rapamycin triggers heterodimerization of FRB/FKBP, leading to irreversible recruitment of pseudojanin (PJ) to the plasma membrane. PJ comprises a 4-phosphatase and 5-phosphatase domain, degrading both $\mathrm{PI}(4,5) \mathrm{P}_{2}$ and $\mathrm{PI}(4) \mathrm{P}$. (e) PLC-mediated DAG production was blocked by recruitment of PJ $(n=16)$,

but not catalytically inactivated PJ (PJ $† ; n=24)$ or PJ with exclusive 5-phosphatase activity (PJ 5Ptase; $n=36$ ). Cells were co-transfected with PKC $\gamma$-C1GFP, M1R, Lyn11-FRB and PJ variants fused to mcherry-FKBP. Cells were selected for membrane recruitment of mcherry. (f) Recruitment of PJ, ( $n=14$ ), but not PJ-5Ptase $(n=9)$ or catalytically inactive PJ $\dagger(n=12)$, suppressed current inhibition by M1R without affecting channel gating by extracellular acidification. Cells were co-transfected with TASK-3, M1R, Lyn11-FRB and PJ variants. Rapamycin $(5 \mu \mathrm{M})$ was applied before the experiments shown. Cells were selected for membrane translocation of mcherry, or for $\mathrm{PJ}$ wt for translocation of co-expressed $\mathrm{PLC} \delta 1-\mathrm{PH}-\mathrm{GFP}$, indicating depletion of $\mathrm{PI}(4,5) \mathrm{P}_{2}$. (g) m-3M3FBS induced translocation of the DAG sensor PKC $\gamma$-C1-GFP, indicating reversible activation of PLC ( $n=20$ cells from three experiments). The inactive analogue o-3M3FBS was largely ineffective ( $n=15$ cells from three experiments). (h) Application of m-3M3FBS ( $n=9)$ but not o-3M3FBS $(n=7)$ reversibly inhibited TASK-3 current. Error bars indicate s.e.m.

turnover by PLC, perhaps because some $\mathrm{PI}(4,5) \mathrm{P}_{2}$ is continuously resynthesized from the augmented $\mathrm{PI}(4) \mathrm{P}$ pool. Alternatively, PI(4)P may itself also be a relevant substrate of PLC $\beta^{20}$. In conclusion, the requirement of $\mathrm{PI}(4,5) \mathrm{P}_{2}$ and $\mathrm{PI}(4) \mathrm{P}$ for TASK channel regulation by GqPCRs but not for basal channel activity corroborates the role of PLC activity in TASK regulation.

We next asked whether PLC activity is also sufficient for inhibiting TASK channels. To this end, we used the benzenesulfonamide $\mathrm{m}$-3M3FBS that activates PLC independent of
$\mathrm{GqPCRs}^{20,32}$. According to plasma membrane translocation of the DAG sensor PKC $\gamma$-C1 m-3M3FBS, but not its inactive analogue o-3M3FBS, activated PLC when applied to $\mathrm{CHO}$ cells (Fig. $2 \mathrm{~g}$ ). The kinetics and amplitude of sensor translocation indicated that activation of PLC by m-3M3FBS occurred more slowly and was somewhat weaker compared with PLC activity triggered by M1R. As shown in Fig. 2h, application of m-3M3FBS robustly inhibited TASK-3 currents, whereas the inactive analogue had little effect. Current inhibition developed slowly, 
was slowly reversible and was less complete compared with receptor activation, mirroring the characteristics of PLC activation as monitored with the fluorescent sensor of PLC activity.

Taken together, these data demonstrate that activation of PLC $\beta$ is an essential requirement for inhibition of TASK-3 channels by a $\mathrm{G}_{\mathrm{q}}$-coupled receptor and that activation of PLC is sufficient to induce channel inhibition. Hence, the signal that mediates channel closure must be downstream of PLC $\beta$.

Diacylglycerol inhibits TASK channels. To identify the relevant biochemical signal, we next directly applied candidate downstream messengers to TASK channels. Application of the membrane permeable short-chain DAG analogue 1,2-Dioctanoyl$s n$-glycerol (DiC8) ${ }^{25}$ to CHO cells expressing TASK-3 or TASK-1 resulted in dose-dependent and reversible current inhibition (Fig. 3a,b). At the same concentration range, DiC8 induced robust but sub-maximal translocation of the DAG sensor domain PKC $\gamma$-C1-GFP to the plasma membrane (Fig. 3c), suggesting that the concentrations reached by exogenous application may be comparable to cellular concentrations of endogenous DAG produced on stimulation of GqPCRs.

As shown in Fig. 3d,e, application of DiC8 to the cytoplasmic face of giant patches excised from Xenopus oocytes similarly inhibited TASK-3 activity. The effectiveness in this cell-free condition points to a direct action of DAG on the channel and argues against an involvement of DAG-dependent protein kinase (PKC) or of DAG metabolites. The inside-out configuration of the giant patch also allowed for the direct delivery of 'native' longchain DAG to the inner leaflet of the membrane (Fig. 3d-f). Endogenous DAG produced by PLC $\beta$ is predominantly 1-stearoyl-2-arachidonoylglycerol $(S A G)^{33}$. Application of SAG $(20 \mu \mathrm{M})$ to giant patches produced a potent inhibition of TASK-3, with an efficacy slightly greater than the short-chain analogue DiC8 at the same concentration (Fig. 3d,e). Channel inhibition was dose-dependent with a half-inhibiting concentration of about $10 \mu \mathrm{M}$ (Fig. 3f). Inhibition was poorly reversible, presumably because partitioning of SAG into the plasma membrane is not readily reversible.

We further tested the lipid metabolites 2-arachidonoyl-glycerol (2-AG) derived from DAG by diacylglycerol lipases (DGLs), and a

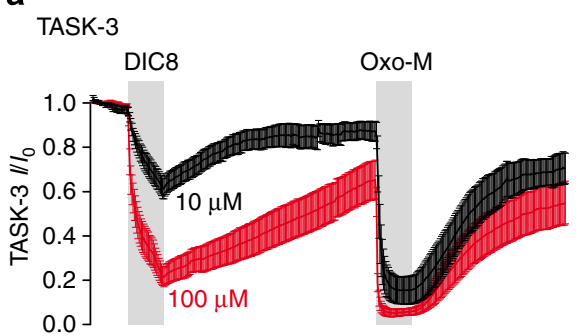

b

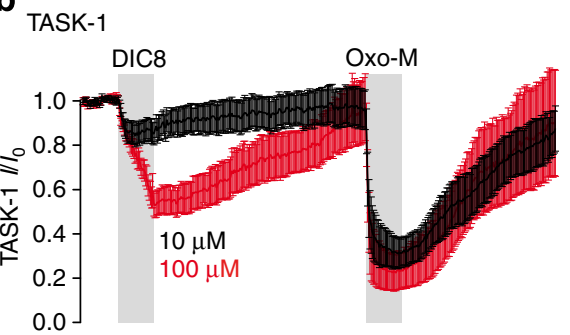

C

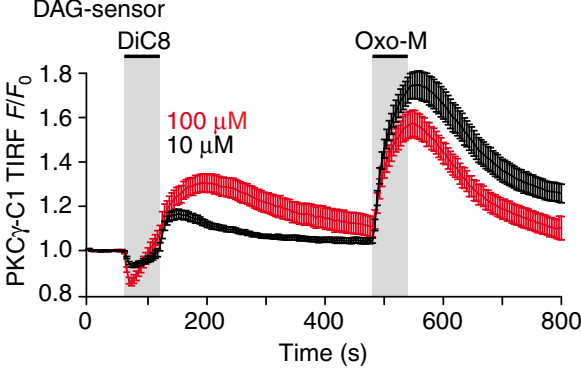

d

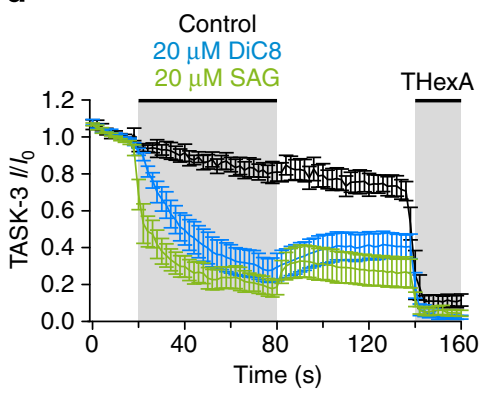

e

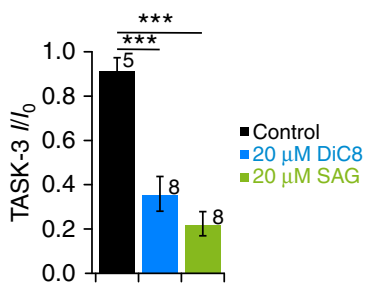

f

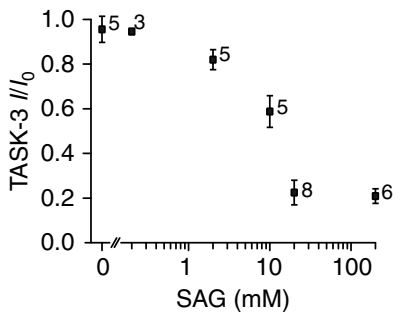

Figure 3 | DAG directly inhibits TASK-1 and $\mathbf{- 3}$ channels. (a) Application of short-chain DAG analogue (1,2-dioctanoyl-sn-glycerol; DiC8) onto CHO cells reversibly inhibited TASK-3; $n=7$ both for application of $10 \mu \mathrm{M}$ (black) and $100 \mu \mathrm{M}$ DiC8 (red). (b) Application of $10 \mu \mathrm{M}(n=8)$ or $100 \mu \mathrm{M}(n=6)$ DiC8 to $\mathrm{CHO}$ cells expressing TASK-1. (c) Membrane association of DAG sensor PKC $\gamma$-C1-GFP induced by application of DiC8 to CHO cells (10 or $100 \mu$ M; $n \geq 29$ cells from $\geq 6$ experiments, each). Activation of the co-expressed M1R by $10 \mu M$ Oxo-M served as a control for strong DAG production.

(d) Application of DiC8 and 1-Stearyl-2-arachidonoyl-sn-glycerol (SAG) inhibited TASK-3 channels when applied to the intracellular face of giant inside-out patches excised from Xenopus oocytes. Tetrahexylammonium (THexA; $10 \mu \mathrm{M}$ ) completely blocking the channel was applied to gauge overall TASK-3 currents. Shown are mean currents measured at $+50 \mathrm{mV}$ at the end of each repetitive voltage ramp, normalized to current size before application $(t=30 \mathrm{~s})$. (e) Residual TASK-3 currents at the end of the DAG application from experiments in (d). ${ }^{\star \star \star}$ indicates $P<0.001$. (f) Dose-dependent inhibition of TASK-3 currents by SAG, measured in giant patches as in $(\mathbf{d}, \mathbf{e})$. Data are presented as mean \pm s.e.m. 
phosphatidic acid (PA) generated by phosphorylation of DAG through diacylglycerol kinases (DGKs) ${ }^{4}$. Consistent with a previous report ${ }^{34}$, the endocannabinoid 2-AG also inhibited TASK-3, albeit with a lower efficacy compared with SAG (Fig. 4a,b). PA or arachidonic acid (AA), which can be released from 2-AG, had little effect on channel activity.

Application of either $\mathrm{IP}_{3}$ or $\mathrm{Ca}^{2}+$ to TASK-3 had no effect on channel activity (Fig. $4 \mathrm{c}, \mathrm{d}$ ), indicating that the $\mathrm{IP}_{3} / \mathrm{Ca}^{2}+$ branch of the PLC-dependent signal-transduction cascade is not involved in TASK regulation by GqPCRs.

DAG dynamics mediate receptor-induced TASK inhibition. These results indicated DAG as a likely messenger mediating TASK channel modulation. We therefore asked whether DAG indeed mediates channel inhibition in response to GqPCR activity. We aimed at altering cellular DAG dynamics and reasoned that accelerating the turnover of DAG should reduce the DAG concentrations reached on receptor stimulation. Cellular DAG accumulating on activation of PLC $\beta$ is cleared by DGKs or by DGLs ${ }^{4}$. Among the 12 isoforms of DGK we found DGK $\beta$ to be constitutively localized at the plasma membrane when overexpressed in $\mathrm{CHO}$ cells (Fig. 5a inset), suggesting that it may effectively turn over DAG after receptor activation. Indeed, expression of DGK $\beta$ strongly blunted the receptor-induced DAG transient in $\mathrm{CHO}$ cells (Fig. 5a,c). When co-expressed with TASK-3 channels, DGK similarly suppressed channel inhibition by activation of M1R (Fig. 5d,f). Overexpression of DAG lipase alpha $\left(\mathrm{DGL} \alpha^{35}\right)$ also strongly attenuated the receptor-induced accumulation of DAG (Fig. 5b,c). As shown in Fig. 5e,f, DGL $\alpha$ also suppressed receptor-mediated channel inhibition

a
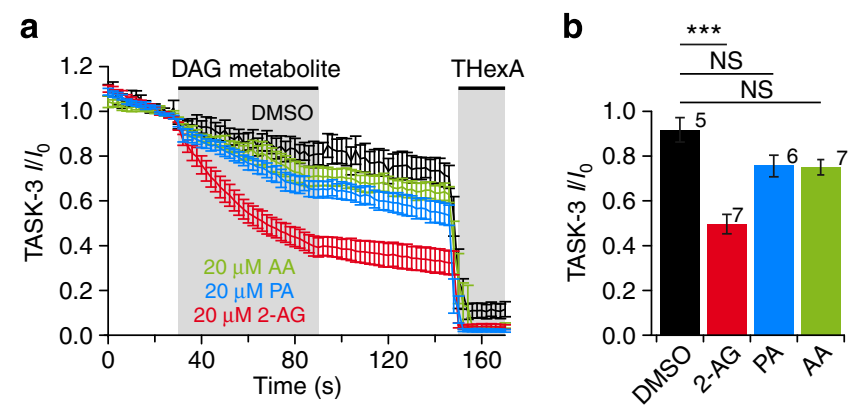

c

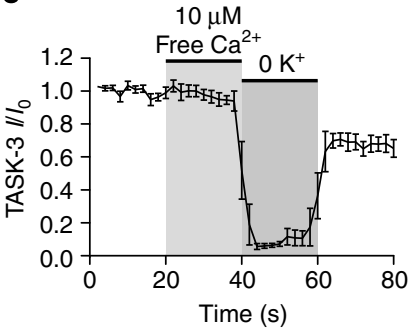

d

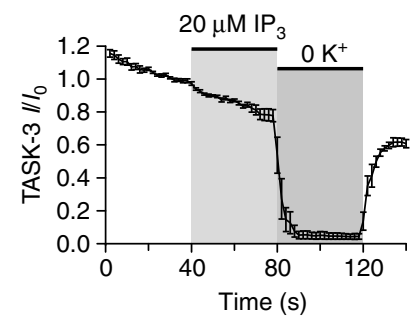

Figure 4 | DAG metabolites, IP 3 and intracellular $\mathrm{Ca}^{2}+$ have little effect on TASK-3 channel activity. (a) Effects of DAG metabolites on TASK-3 currents in giant inside-out patches from Xenopus oocytes. Control data with application of vehicle (DMSO) is replotted from Fig. 3d for comparison. (b) Mean residual TASK-3 currents measured at the end of the application of the various lipids from experiments in (a). (c) Application of $10 \mu \mathrm{M}$ free $\mathrm{Ca}^{2}+$ to the intracellular face of giant patches was without effect on TASK3 currents $(n=6)$. Currents measured as in (a). Subsequent removal of intracellular $\mathrm{K}^{+}$completely eliminated $\mathrm{K}^{+}$outward currents, verifying that currents were mediated by TASK-3. (d) Application of $20 \mu \mathrm{MIP} 3(n=7)$ to inside-out patches did not inhibit TASK-3 currents. Giants patch recordings were done as in (a) and (c). Data are presented as mean \pm s.e.m. and accelerated current recovery when co-expressed with TASK-3 channels.

These findings are consistent with DAG acting as the endogenous messenger that inhibits TASK channels. At the same time, the results rule out a major role of downstream lipid messengers in inhibiting TASK channels, including PA and 2-AG and its metabolites, as their concentrations should be increased rather than decreased by DGK and DGL, respectively. We note that the impairment of current inhibition by DGL $\alpha$ appears less pronounced than the decrease in the DAG transient reported by the PKC $\gamma$-C1-GFP sensor. However, the remaining channel inhibition may be readily explained by weak inhibition of TASK by the DGL product 2-AG, as observed in isolated patches (Fig. 4a).

A proximal C-terminal motif essential for inhibition by DAG. A six-amino-acid motif (VLRFLT) located at the proximal C terminus of TASK-3 channels is essential for the channels' sensitivity to GqPCR modulation ${ }^{14}$. If DAG mediates the receptor's effect, the same motif must also determine the sensitivity to exogenous DAG. We therefore next examined TASK channels with mutation or deletion of the short motif (Fig. 6a). Figure $6 \mathrm{~b}$ shows that truncation of the $\mathrm{C}$ terminus of TASK-3 preserving the VLRFLT motif $(\Delta 249)$ yielded functional channels still sensitive to regulation by GqPCRs, consistent with previous findings ${ }^{14}$. However, additional removal of the VLRFLT motif $(\Delta 244)$ rendered the channel insensitive to activation of the co-expressed receptor. These channels were still gated by extracellular $\mathrm{pH}$, indicating that the fundamental function of the truncated channels was not compromised. Strikingly, the sensitivity of these truncated channels to DAG mirrored their inhibition by the GqPCR. Thus, inhibition by DiC8 $(20 \mu \mathrm{M})$ was unchanged in $\Delta 249$, but was abrogated by truncation of the VLRFLT motif (Fig. 6c).

We further analysed the relevance of this motif by replacing individual amino acids by alanine. As shown in Fig. 6d, these mutations impaired GqPCR-dependent regulation to different degrees, with the most pronounced effects observed in the L244A and R245A mutants. When challenged with DiC8, the resulting channel inhibition of L244A and R245A was also largely reduced (Fig. 6e). Overall, inhibition by DiC8 of the various mutant channels correlated well with their sensitivity to receptor activity (Fig. 6f).

In conclusion, inhibition of TASK channels by DAG relies on the same molecular determinants that govern inhibition by Gq signalling, lending further support to the conclusion that receptor/Gq-induced TASK channel inhibition is mediated by increased DAG levels.

DAG inhibits TASK currents in cerebellar granule neurons. Regulation of TASK channels by Gq-coupled receptors strongly impacts on membrane potential and excitability in many cell types. In cerebellar granule neurons (CGNs), a large 'standing outward' current $\left(\mathrm{IK}_{\mathrm{SO}}\right)$ dominated by TASK-1 and TASK-3 channels ${ }^{36-38}$ controls resting potential and action potential firing $8,36,38$. Gq-coupled neurotransmitter receptors (including muscarinic acetylcholine receptors and metabotropic glutamate receptors) dynamically regulate CGN excitability by inhibition of TASK channels ${ }^{12,39}$.

We examined the activity of short-chain DAG, DiC8, on $\mathrm{IK}_{\mathrm{SO}}$ in dissociated cultured CGNs. Application of DiC8 $(100 \mu \mathrm{M})$ reduced the outward current by about $50 \%$, similar to current suppression by extracellular acidification or activation of endogenous muscarinic acetylcholine receptors (Fig. 7a,b). As CGNs express other K2P channels in addition to $\mathrm{TASK}^{40}$, we combined extracellular acidification with application of DAG 
a

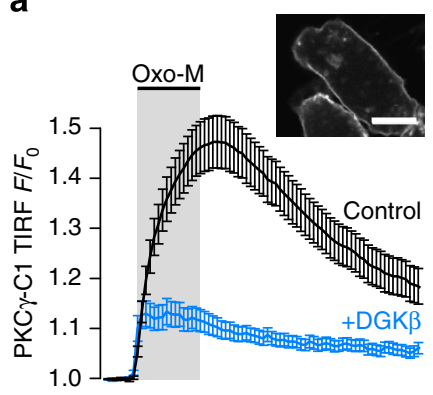

c

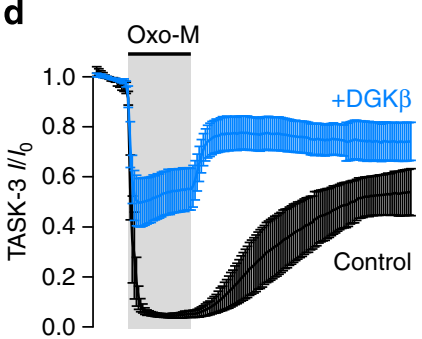

b

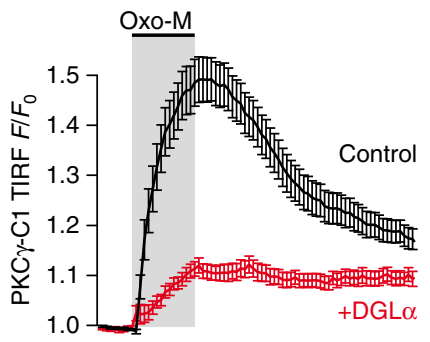

e

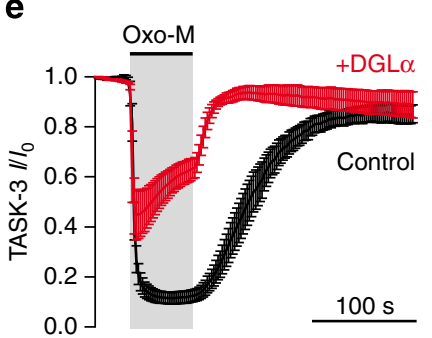

C

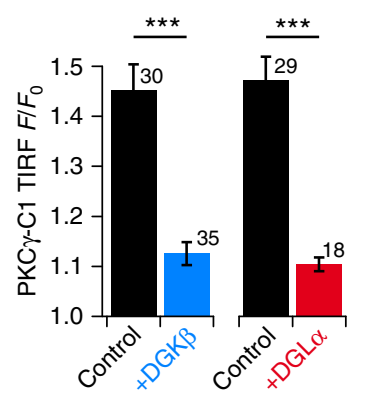

f

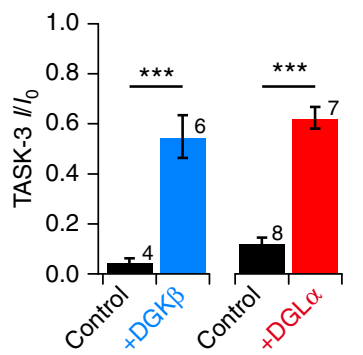

Figure 5 | Suppression of cellular DAG-transients attenuates receptor-mediated TASK-3 inhibition. (a) DAG concentration dynamics in CHO cells induced by M1R (10 $\mu \mathrm{M}$ Oxo-M) were measured by TIRF microscopy of PKC $\gamma$-C1-GFP either in the absence (control) or with co-expression of DAG-kinase $\beta$ (DGK $\beta$ ). Confocal image (inset) shows membrane localization of GFP-tagged DGK $\beta$ (Scale bar, $10 \mu \mathrm{m}$ ). (b) Receptor-induced DAG dynamics measured as in (a) either without (control) or with co-expression of DAG lipase $\alpha$ (DGL $\alpha$ ). (c) Mean TIRF signal increase ( \pm s.e.m.) at the end of $60 \mathrm{~s}$ Oxo-M application from experiments shown in (a) and (b). Numbers indicate individual recordings. (d) TASK-3 current inhibition by activation of M1R with or without co-expression of DGK $\beta$. (e) TASK-3 current inhibition by activation of M1R with or without co-expression of DGL $\alpha$. (f) Mean residual TASK-3 currents at the end of Oxo-M application from the experiments shown in (d,e). Current inhibition was strongly reduced by either DGK $\beta$ or DGL $\alpha$, matching the suppressed DAG transients (a-c).

to test whether the DAG-sensitive currents correspond to neuronal TASK channels. As shown in Fig. 7c, only a minor fraction of residual $\mathrm{IK}_{\mathrm{SO}}$ remained sensitive to DAG when TASK currents were blocked by $\mathrm{pH} 5.9$ and acid-sensitive TASK currents remained closed when switching back to $\mathrm{pH} 7.4$ after exposure to DAG. These observations support the conclusion that most of the DAG-sensitive currents in CGNs was indeed mediated by TASK. Taken together, these findings suggest that the current component carried by TASK channels is sensitive to DAG, consistent with the idea that the inhibition of native TASK channels by neuronal GqPCRs is indeed mediated by DAG.

Application of DAG also depolarized the membrane potential of CGNs by about $10 \mathrm{mV}$ (Fig. 7d,e), similar to activation of endogenous ACh receptors, indicating that DAG-mediated regulation of TASK channels underlies modulation of neuronal electrical excitability.

\section{Discussion}

Here, we show that GqPCRs inhibit TASK channels through the production of DAG. Thus, several independent assays indicated that PLC activity is essential for channel inhibition. Among the downstream messengers of PLC, only DAG induced channel inhibition. Moreover, accelerating the turnover of DAG by overexpression of either DGK or DGL suppressed channel inhibition, demonstrating that liberation of DAG is indeed necessary for channel regulation.

TASK channels are inhibited by DAG and not through messengers downstream of DAG, as DAG application rapidly inhibited channels in a cell-free system (excised patch), where recruitment of cytoplasmic DAG effectors, activation of kinase signalling, or conversion of DAG to downstream messengers is unlikely. In principle DAG could act on TASK channels via activation of protein kinase C (PKC), as pharmacological activation PKC can modulate TASK channels ${ }^{41}$. However, several careful studies consistently found that PKC does not mediate GqPCR-induced inhibition. Thus, neither removal of all potential PKC phosphorylation sites ${ }^{14,41}$ nor pharmacological blockade of PKC ${ }^{12,19,22,41}$ affected receptor-dependent channel regulation. This is in line with our current results showing that DAG is effective in isolated patches and inhibits truncated channels lacking potential PKC phosphorylation sites.

The model that follows is direct binding of DAG to the channel. This could be binding of DAG directly either to the pore-forming $\alpha$-subunits or to an accessory protein that is present in the plasma membrane and interacts with the $\alpha$-subunit. We consider direct binding to the channel as the more simple and straightforward model, since (i) to our knowledge, appropriate interaction partners of TASK-1/3 are not known, (ii) the C-terminally truncated TASK channel should be deprived of much of its potential cytoplasmic protein-protein interaction interfaces and (iii) a relevant accessory protein would have to be ubiquitously and abundantly expressed in plasma membranes as DAG was effective in a wide variety of cells ranging from Xenopus oocytes to neurons; in fact, GPCR-mediated TASK channel inhibition is reported to work in essentially any cell type examined.

As enzymatic conversion of DAG into PA or 2-AG reduced channel inhibition, these downstream intermediates cannot mediate channel inhibition. It is worth noting, however, that in the excised patch, 2-AG also inhibited TASK channels and that increased conversion of DAG to 2-AG did not fully block 
a
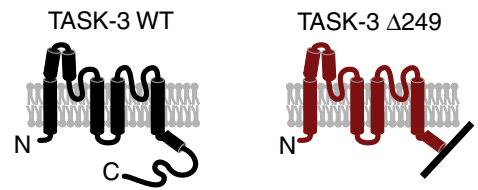

b

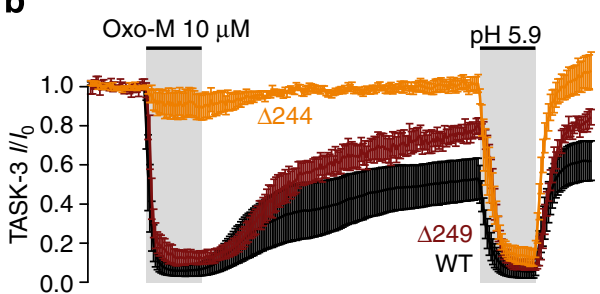

d

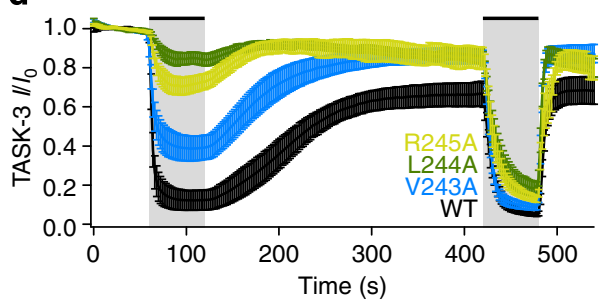

f

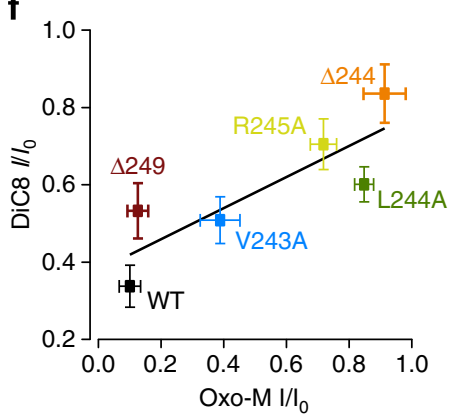

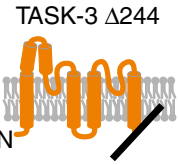

C

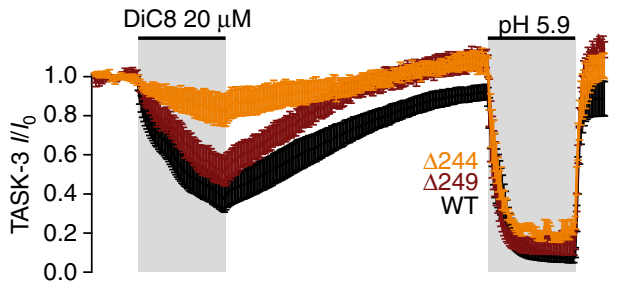

e

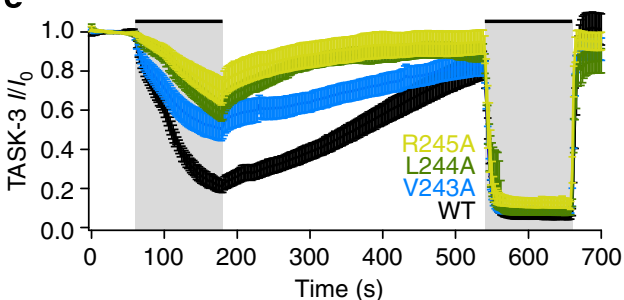

Figure 6 | The C-terminal VLRFLT motif is critical for DAG-mediated channel inhibition. (a) Schematic representation of C-terminal channel truncations: TASK-3 was truncated either after position 248, retaining the VLRFLT motif, (TASK-3 4249 ) or after position 243, removing the entire C terminus (TASK-3 4244 ). (b) Removal of the VLRFLT motif rendered TASK-3 currents insensitive to co-expressed $\mathrm{G}_{\mathrm{q}}$-coupled M1R, whereas truncation after this motif had no effect on channel regulation ( $n=6,7$ and 5 cells, for wt, $\Delta 249$, and $\Delta 244$ channels, respectively). Inhibition by extracellular acidification demonstrated that currents were carried by TASK-3 and confirmed functional integrity of truncated channels. (c) Whole-cell recordings of the effect of DiC8 on TASK-3 and truncated channels ( $n=9,9$ and 7 cells, for wt, $\Delta 249$ and $\Delta 244$ channels, respectively) expressed in CHO cells. (d) Point mutations in the VLRFLT motif reduced the channel sensitivity towards M1R-mediated inhibition ( $n=11,10,10,11$ cells for wt, V243A, L244A and R245A, respectively). (e) Channel mutants with attenuated receptor-induced inhibition also showed reduced sensitivity towards application of $20 \mu \mathrm{M}$ DiC 8 ( $n=5,7,7$ and 5 cells for wt, V243A, L244A and R245A, respectively). (f) Correlation of channel inhibition by M1R receptor versus application of $\mathrm{DiC} 8$, derived from the experiments presented in (b-e). Shown are normalized current amplitudes at the end of the application of Oxo-M or DiC8 (mean \pm s.e.m.).

receptor-induced channel inhibition, although the DAG transient was strongly attenuated. Thus, production of 2 -AG may also make a minor contribution to TASK channel regulation by GqPCRs.

It was proposed previously that TASK inhibition is mediated by direct interaction with activated $\mathrm{G} \alpha_{\mathrm{q}}$ rather than by downstream signals ${ }^{18}$. In particular a direct biochemical interaction between $G \alpha_{\mathrm{q}}$ and the TASK channel supports such a mechanism. However, as the various independent approaches blocking PLC function all abolished receptor-dependent inhibition it seems highly unlikely that $\mathrm{G} \alpha_{q}$ alone could be sufficient to inhibit TASK channels. On the contrary, DAG by itself is sufficient to downregulate the channels. Yet it remains possible that $G \alpha_{q}$ could contribute to channel inhibition by increasing DAG affinity or efficacy through direct TASK-G $\alpha_{\mathrm{q}}$ interaction, although such a model is speculative at present.
Although the control of TASK-1/3 by DAG has not been recognized before, it is well established that other lipid compounds inhibit these channels. The endocannabinoid anandamide and its synthetic analogue methanandamide potently inhibit TASK channels by direct interaction with the channel ${ }^{34,42}$. Our current finding of partial inhibition by 2-AG suggests another endocannabinoid as a regulator of these background channels. Notably, all these compounds are chemically similar to DAG in consisting of arachidonic acid bound to a small moderately polar headgroup containing a hydroxyl group. It thus seems likely that all of these compounds share a common mechanism of TASK inhibition. It is further interesting to note that sanshool, the pungent compound of Szechuan pepper, acts by inhibiting TASK channels ${ }^{43}$. Sanshool is chemically similar to the aforementioned compounds in being an amide of a 
a

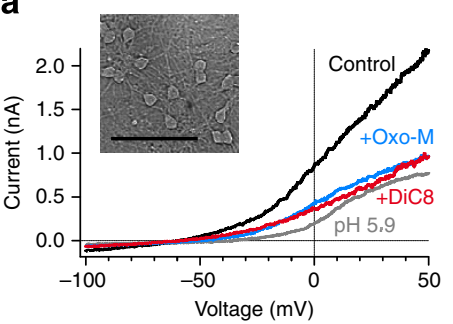

b

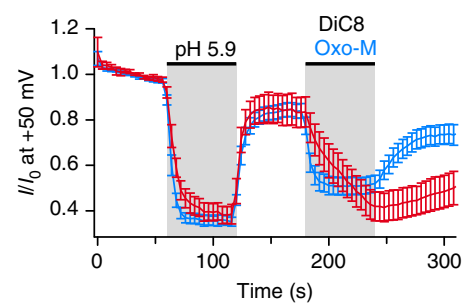

C

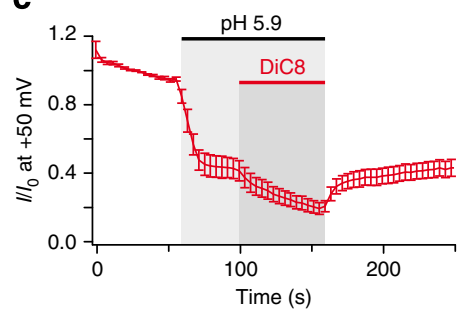

d

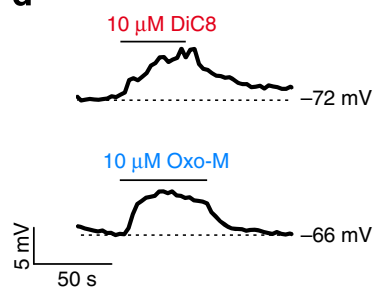

e

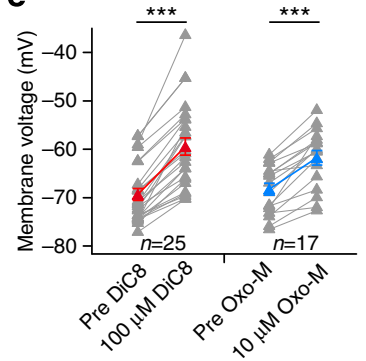

Figure $\mathbf{7}$ | Inhibition of native $\mathbf{I K}_{\mathrm{so}}$ in cerebellar granule cells by DAG.

(a) Representative whole-cell currents from a dissociated cerebellar granule cell (inset; scale bar, $50 \mu \mathrm{m}$ ). Currents were recorded in response to voltage ramps from a holding potential of $+50 \mathrm{mV}$. Application of $10 \mu \mathrm{M}$ Oxo-M to activate endogenous muscarinic receptors or $100 \mu \mathrm{M}$ DiC8 inhibited outward potassium currents to a similar extent as extracellular acidification to $\mathrm{pH}$ 5.9. (b) Mean time course of standing outward currents during application of $10 \mu \mathrm{M}$ Oxo-M $(n=18)$ or $100 \mu \mathrm{M} \mathrm{DiC} 8(n=7)$, and extracellular acidification. Currents were measured at $+50 \mathrm{mV}$ and normalized to current amplitude before initial acidification. (Error bars indicate s.e.m.). (c) Mean time course of $\mathrm{I}_{\mathrm{SO}}$ in response to combined application of low extracellular pH and DiC8 $(100 \mu \mathrm{M})$. (d) Representative recordings of membrane potential during application of DiC8 (upper trace) or Oxo-M (lower trace). (e) Change of membrane potential induced by application of DiC8 $\left(\Delta V_{\mathrm{m}}=9.9 \pm 0.9 \mathrm{mV}\right)$ or muscarinic receptor stimulation $\left(\Delta V_{\mathrm{m}}=6.5 \pm 0.8 \mathrm{mV}\right)$ from experiments performed as in (d).

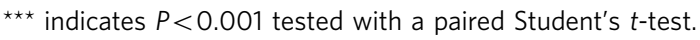

polyunsaturated fatty acid and a moderately polar alcohol. It is tempting to speculate that sanshool elicits sensory effects by hijacking a binding site at TASK channels built for regulation via endogenous lipid messengers.

All well-characterized DAG effectors are earmarked by containing at least one prototypical C1 DAG-binding domain. Among the C1-containing proteins, the PKCs are often considered as the canonical and most important DAG effectors. Additional protein families containing $\mathrm{Cl}$ domains are the chimaerins, DGKs, protein kinase D and Munc13s $\mathrm{s}^{1,4}$. In contrast, TASK channels do not contain a C1 domain. DAG also contributes to the activation of certain canonical TRP (TRPC) channels ${ }^{6,44}$, which also lack a C1 domain. To our knowledge, the binding site for DAG has not been identified.

Our data show that a six-amino-acid motif (VLRFLT) at the most proximal intracellular C terminus of TASK-3 is crucial for DAG inhibition. Although its location close to the membrane's

inner leaflet may suggest a role in DAG binding, the membraneproximal $\mathrm{C}$ terminus is implicated in gating of other $\mathrm{K} 2 \mathrm{P}$ channels by a variety of stimuli. In TREK channels it mediates channel gating by phospholipids, membrane tension, intracellular $\mathrm{pH}$ and the antidepressant fluoxetine ${ }^{45-47}$. In TASK channels, the VLRFLT motif is also essential for channel activation by volatile anaesthetics ${ }^{14}$. Thus, it seems that the VLRFLT motif is most likely a gating element coupling DAG binding to channel closure; however, it is also possible that it contributes to binding of DAG. Yet, it remains to be shown if DAG indeed binds directly to the channel's pore-forming subunit. If so, the fact that even the truncated minimalistic channel remains sensitive to DAG should facilitate identification of the DAG binding sites in TASK channels; the only intracellular structures to be considered as potential interaction sites of the headgroup of membraneembedded DAG remaining in truncated TASK channel are the VLRFLT motif, a short $\mathrm{N}$ terminus of seven amino acids, and an intracellular loop of some 30 amino acids connecting M2 and M3. Thus, TASK channels may be amenable to the study of structural details of DAG binding and may serve as a novel paradigm for DAG effector molecules not operated via $\mathrm{C} 1$ domains.

How might DAG alter gating to close the channels? Ashmole et al. ${ }^{48}$ hypothesize that the underlying gating mechanism may be related to the gating by voltage, where depolarization increases open probability. Future single channel studies may provide more insights into the mechanistic details.

Our results establish the control of K2P channels as a novel signalling role for DAG. Given the widespread expression of TASK channels and their established functions ${ }^{7}$, for example, in neurons ${ }^{9,15,38}$ and adrenocortical cells ${ }^{17}$, this new signalling pathway is most likely important in many physiological processes. The tight regulation of plasma membrane DAG concentrations may be critical for maintaining appropriate TASK channel activity. Specifically, DAG concentration dynamics triggered by PLC activation depend on the rate of DAG clearance. Among the 10 mammalian DGK isoforms, several have been associated with the control and termination of PLC/DAG signalling in the plasma membrane ${ }^{4,49}$. Interestingly, mutation of $\mathrm{DGK} \delta$, one of DGK isoforms prominently expressed in the brain, is associated with epilepsy in humans and mice ${ }^{50}$. In principle, such a phenotype might result from excessive suppression of a potassium conductance by increased DAG levels. Knock-out of other neuronal DGKs also leads to neuronal and behavioural alterations that are linked to changed DAG signalling ${ }^{51,52}$. It should be interesting to examine whether dysregulation of TASK channel activity plays a role in these pathologies. DAG is also turned over by DGLs to generate the endocannabinoid 2-AG. Although their signalling function has been considered mostly in terms of production of the signalling lipids $2-\mathrm{AG}$ and $\mathrm{AA}^{35}$, a role in regulating TASK channels by curtailing DAG signals should be considered, given that experimental overexpression of DGL $\alpha$ strongly affected DAG transients (Fig. 5).

In summary, our findings indicate that DAG dynamics and homeostasis directly control a multitude of cellular processes dependent on membrane potential, such as neuronal excitability, muscle contraction and hormone secretion through the inhibition of TASK-1 and -3 channels.

\section{Methods}

Cell culture. $\mathrm{CHO} \mathrm{dhFr}^{-}$cells were grown at $37^{\circ} \mathrm{C}$ with $5 \% \mathrm{CO}_{2}$ in minimum essential medium (MEM)- $\alpha$ with nucleosides supplemented with $10 \%$ fetal calf serum (Biochrom AG, Berlin, Germany), $100 \mathrm{U} \mathrm{ml}^{-1}$ penicillin and $100 \mu \mathrm{g} \mathrm{ml}^{-1}$ streptomycin. $\mathrm{G} \alpha_{\mathrm{q} / 11}-1-$ fibroblasts (kindly provided by Douglas A. Bayliss) were cultured at $37^{\circ} \mathrm{C}$ with $5 \% \mathrm{CO}_{2}$ in Dulbecco's modified eagle medium (DMEM) with high glucose and GlutaMax supplemented with $10 \%$ fetal calf serum (Biochrom AG), $100 \mathrm{U} \mathrm{ml}^{-1}$ penicillin and $100 \mathrm{\mu g} \mathrm{ml}^{-1}$ streptomycin. Both cell lines were seeded onto glass coverslips or glass-bottom dishes (WillCo Wells B.V., 
Amsterdam, The Netherlands) for electrophysiology and imaging experiments, respectively. One to two days after seeding cells were transfected using JetPEI (Polyplus Transfection, Illkirch, France) according to the manufacturer's protocol. Experiments were performed $24-72 \mathrm{~h}$ post transfection.

CGNs were prepared from P6-P9 Wistar rats. Animals were anaesthetized and decapitated to obtain cerebella, which were washed in ice-cold PBS, cut in 3-4 pieces and incubated at $37^{\circ} \mathrm{C}$ for $15 \mathrm{~min}$ in $0.05 \%$ trypsin-EDTA/HBSS. After washing with DMEM, cerebelli were incubated with $10 \%$ DNase I (Sigma-Aldrich, Taufkirchen, Germany)/DMEM at $37^{\circ} \mathrm{C}$ for $4 \mathrm{~min}$. Cells were then triturated using fire-polished glass Pasteur pipettes with decreasing openings, passed through a $70-\mu \mathrm{m}$ cell strainer and washed in a centrifugation step of $10 \mathrm{~min}$ at $210 \mathrm{~g}$ at $4{ }^{\circ} \mathrm{C}$. CGNs were resuspended in DMEM with high glucose and GlutaMax supplemented with $10 \%$ horse serum (Biochrom AG), $100 \mathrm{U} \mathrm{ml}^{-1}$ penicillin and $100 \mu \mathrm{g} \mathrm{ml}^{-1}$ streptomycin and $25 \mathrm{mM} \mathrm{KCl}$ and plated on poly-D-lysine coated (MW 70,000150,000; Sigma-Aldrich) glass coverslips with a density of 400,000 cells per $\mathrm{cm}^{2}$. After $1 \mathrm{~h}$ of cell sedimentation, $1 \mu \mathrm{M}$ cytosine $\beta$-D-arabinofuranoside (SigmaAldrich) was added to the medium to prevent glial growth. Neurons were used for whole-cell recordings after 7-12 days in culture.

Media and buffers used for cell culture were obtained from Gibco (Life Technologies, Carlsbad, CA, USA) unless noted otherwise.

Molecular biology. The following expression plasmids were used for transient transfection of CHO cells or $\mathrm{G}_{\mathrm{q} / 11}{ }^{-}$- fibroblasts: rat PKC $\gamma-\mathrm{Cl}$ (AA 26-89 in pEGFP-N1 NM_012628.1; obtained from Tobias Meyer); human PLC81-PH (AA 1-170 in pEGFP-N1; NM_006225.3, obtained from Tamas Balla); mouse $\mathrm{G} \alpha_{\mathrm{q}}$ (in pcDNA3, CFP inserted between AA 124/125 (ref. 53); NM_008139.5); mutant $G \alpha_{q}$ constructs $\mathrm{G} \alpha_{\mathrm{q}}-\mathrm{AA}$ and $\mathrm{G} \alpha_{\mathrm{q}}-5 \mathrm{~A}$ contain the point mutations R256A/T257A or DNE243-245AAA/R256A/T257A, respectively; human G $\alpha_{\text {qiq }}$ (chimera of G $\alpha$ q AA 1-245 + AA 333-359; NM_002072.4 and Gai AA 242-328; NM_002070.3 in pcDNA3.1; kind gift from Xuming Zhang); human TASK-1 (in pcDNA3.1; NM_002246.2), human TASK-3 (in pcDNA3.1; NM_001282534.1; obtained from Jürgen Daut); human M1 receptor (in pSGHV0; NM_000738.2), rat DGK $\beta$ (in pEGFP-C3, GFP was removed for TIRF experiments; NM_019304.1, kindly provided by Fumio Sakane); human DGL $\alpha$-GFP (in pcDNA3.1/V5-His TOPO; NM_006133.2, gift from Patrick Doherty); mcherry-FKBP-Pseudojanin (in pEGFP-C1; AA 30-108 of FKBP NM_054014.3, AA 2-517 of SAC1

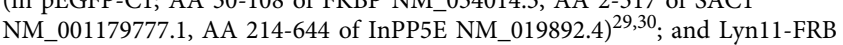
(in pC4RHE, AA 1-11 of Lyn11 (GCIKSKGKDSAGA), AA 2026-2121 of FRB NM_004958.3). Mutations of $G \alpha_{\mathrm{q}}$, hTASK-3, Pseudojanin (C392S of original $\mathrm{SAC} 1$ ) and C-terminal truncations of hTASK-3 were generated using the QuikChange II XL Site-Directed mutagenesis kit (Stratagene, Agilent Technologies, Waldbronn, Germany). For expression in oocytes, human TASK-3 in pSGEM was used. YFP was fused to the $\mathrm{N}$ terminus of human PLC $\beta 3$ via a 10 AS flexible linker by means of gateway cloning, and the resulting cDNA was cloned into pcDNA3. All constructs were verified by sequencing.

Chemicals. DAG analogues and metabolites (Avanti Polar Lipids, Alabaster, AL, USA) were prepared as $20 \mathrm{mM}$ stock in DMSO. PLC inhibitor U73122 and the inactive analogue U73343 (Sigma-Aldrich) were prepared as $5 \mathrm{mM}$ stock solution in DMSO, kept at $-20^{\circ} \mathrm{C}$ and diluted into physiological solutions $<1 \mathrm{~h}$ before experiments. The PLC activator m-3M3FBS and its inactive analogue o-3M3FBS (Tocris Bioscience, Bristol, United Kingdom) were prepared as $50 \mathrm{mM}$ stocks in DMSO. Muscarinic agonist Oxotremorine M (Oxo-M; Tocris Bioscience) was dissolved as $10 \mathrm{mM}$ stock in water. Rapamycin was purchased as $5 \mathrm{mM}$ solution in DMSO (Calbiochem, Merck Chemicals GmbH, Schwalbach, Germany). All drugs were stored at $-20^{\circ} \mathrm{C}$ and diluted in extracellular solution directly before usage. Inositol-1,4,5-trisphosphate ( $\mathrm{IP}_{3}$; Sigma-Aldrich) was prepared as $5 \mathrm{mM}$ in water and diluted in intracellular solution for excised patch measurements.

Patch-clamp. Whole-cell voltage clamp experiments on $\mathrm{CHO} \mathrm{dhFr}^{-}, \mathrm{G}_{\mathrm{q} / 11}{ }^{-1-}$ fibroblasts or primary CGNs were performed with an Axopatch 200B amplifier (Molecular Devices, Sunnyvale, CA, USA) controlled by PatchMaster v2x41 software (HEKA, Lambrecht/Pfalz, Germany) via an ITC-16 interface (InstruTECH, HEKA). Currents were low-pass filtered at $2 \mathrm{kHz}$ and sampled at $5 \mathrm{kHz}$.

Patch pipettes were pulled with a Sutter P-2000 (Sutter Instruments, Novato, CA, USA) from borosilicate glass (Science Products, Hofheim, Germany) to an open pipette resistance of 1.5 to $3 \mathrm{M} \Omega$ when filled with pipette solution containing (mM) $135 \mathrm{KCl}, 5$ Hepes, 5 EGTA, $3.5 \mathrm{MgCl}_{2}, 2.5 \mathrm{Na}_{2} \mathrm{ATP}, 2.41 \mathrm{CaCl}_{2}$ and 0.1 $\mathrm{Na}_{2} \mathrm{GTP}$, adjusted to pH 7.3 with KOH. To buffer intracellular $\mathrm{Ca}^{2+}$ to effectively zero, the solutions contained (in mM) $85 \mathrm{KCl}, 5$ Hepes, $20 \mathrm{~K}_{4}$ EGTA or $\mathrm{K}_{4} \mathrm{BAPTA}$, $1 \mathrm{MgCl}_{2}, 2.5 \mathrm{Na}_{2} \mathrm{ATP}$ and $0.1 \mathrm{Na}_{2} \mathrm{GTP}$. Alternatively, intracellular solution contained $5 \mathrm{mM} \mathrm{K}_{4} \mathrm{BAPTA}$ with $\mathrm{KCl}$ changed to $130 \mathrm{mM}$. Whole-cell serial resistance was $<8 \mathrm{M} \Omega$, which was compensated by $60 \%$. Extracellular solution was composed of (mM) $144 \mathrm{NaCl}, 10 \mathrm{HEPES}, 5.8 \mathrm{KCl}$, 5.6 glucose, $1.3 \mathrm{CaCl}_{2}, 0.9$ $\mathrm{MgCl}_{2}, 0.7 \mathrm{NaH}_{2} \mathrm{PO}_{4}$ and set to $\mathrm{pH} 7.4$ or pH 8.4 (for TASK-1) or pH 5.9 (to block TASK currents) with $\mathrm{NaOH}$. Owing to the development of a cationic current during application of U73122, the extracellular $\mathrm{Na}^{+}$was substituted with $\mathrm{NMDG}^{+}$in the respective recordings.
Giant patch recordings from Xenopus laevis oocytes were performed as described previously ${ }^{54}$. In brief, cRNA was synthesized using the mMESSAGE mMACHINE Kit (Ambion, Life Technologies). Xenopus laevis oocytes were obtained from EcoCyte Bioscience (Castrop-Rauxel, Germany). Following injection of cRNA, oocytes were incubated at $18^{\circ} \mathrm{C}$ for $1-3$ days before performing giant patch recordings. Currents were recorded using an EPC10 amplifier (HEKA) controlled by PatchMaster (HEKA), low-pass filtered at $2.9 \mathrm{kHz}$ and sampled at $5 \mathrm{kHz}$. Giant patch pipettes were fire-polished to obtain open pipette resistances of $0.2-0.5 \mathrm{M} \Omega$ when filled with extracellular solution containing (mM) $115 \mathrm{NaCl}, 10$ HEPES, $5 \mathrm{KCl}, 1.5 \mathrm{CaCl}_{2}, 1 \mathrm{MgCl}_{2}$, adjusted to $\mathrm{pH} 7.4$ with $\mathrm{NaOH}$. Patches were excised into intracellular bath solution containing (in mM) $120 \mathrm{KCl}, 5$ HEPES, 3.5 $\mathrm{MgCl}_{2}, 1 \mathrm{~K}_{2}$ EGTA and was set to $\mathrm{pH} 7.2$ with $\mathrm{KOH}$. Intracellular solutions were applied to the cytoplasmic face of the patch with a multi-barrel gravity-driven application system. For $\mathrm{K}^{+}$-free intracellular solution $120 \mathrm{mM} \mathrm{NaCl}$ was substituted for $\mathrm{KCl}$. For application of intracellular $\mathrm{Ca}^{2+}$, the $\mathrm{K}_{2}$ EGTA concentration was reduced to $2 \mathrm{mM}$ and $1.98 \mathrm{mM} \mathrm{Ca}^{2+}$ was added, yielding $10 \mu \mathrm{M}$ free $\mathrm{Ca}^{2+}$. In these experiments chloride was substituted by methanesulfonate to minimize currents through $\mathrm{Ca}^{2+}$-activated chloride channels.

All recordings were performed at room temperature $\left(\sim 21^{\circ} \mathrm{C}\right)$. TASK currents were recorded in response to voltage ramps $(-100 \mathrm{mV}$ to $+50 \mathrm{mV}$ over $500 \mathrm{~ms})$ followed by $200 \mathrm{~ms}$ at $+50 \mathrm{mV}$ and repeated every $2 \mathrm{~s}$. To quantify current size and time course of current amplitude, currents at $+50 \mathrm{mV}$ were averaged for each trace and normalized to the initial current $I_{0}$ before drug application. In cerebellar granule cells, the standing outward current, $\mathrm{IK}_{\mathrm{SO}}$, was measured at $+50 \mathrm{mV}$ after $2.5 \mathrm{~s}$ of depolarization to minimize currents through voltage-dependent $\mathrm{K}^{+}$ channels by inactivation.

TIRF microscopy. TIRF imaging was performed as previously described ${ }^{55}$. In brief, we used a BX51WI upright microscope equipped with a TIRF-condenser (Olympus, Hamburg, Germany), directing a 488-nm laser to obtain total reflection. Fluorescence images were acquired every $4 \mathrm{~s}$ with a 530 -nm-long-pass emission filter and a CCD camera controlled by TillvisION software (TILL Photonics $\mathrm{GmbH}$, Gräfelfing, Germany). Mean fluorescence intensity $F$ of a single region of interest chosen to cover the majority of the cell's footprint was background subtracted and normalized to the averaged intensity $F_{0}$ at the beginning of the experiment or immediately before application of the receptor agonist.

FRET microscopy. Fluorescence resonance energy transfer between $\mathrm{G} \alpha_{\mathrm{q}}$-CFP and YFP-PLC $\beta 3$ was measured essentially as described ${ }^{53}$. In brief, $\mathrm{CHO}$ cells were transiently transfected with G $\alpha_{\mathrm{q}}$-CFP, YFP-PLC $\beta 3, \mathrm{M} 1 \mathrm{R}$, as well as G $\beta_{1}$ and $\mathrm{G} \gamma_{2}$, to maintain stoichiometry of heterotrimeric G-proteins ${ }^{53}$. Single whole-cell patch-clamped cells were imaged on a Nikon Eclipse TE2000-U inverted microscope equipped with a Nikon Plan Apo VC 60x/1.40 Oil $\infty / 0.17$ DIC N2 WD 0.13 oil-immersion objective (Nikon GmbH, Düsseldorf, Germany). To avoid fluorophore (YFP) quenching, intracellular chloride was reduced to $60 \mathrm{mM}$ in these experiments with $\mathrm{KCl}$ replaced by equimolar $\mathrm{K}$-Aspartate.

The fluorophores were excited using an Oligochrome (Till Photonics, Gräfelfing, Germany), and fluorescence intensities were acquired with fluorescence detection unit (FDU) photodiodes and Oligochrome Imagining Control Unit (ICU FDU-2) (TILL Photonics). CFP was excited through a 430/24 ET bandpass filter. Fluorescence emission intensities were collected using a beam splitter with a 480/40m ET bandpass for CFP $\left(\mathrm{F}_{480}\right)$ and a 535/30m ET bandpass filter for YFP $\left(\mathrm{F}_{535}\right)$ (all filters from AHF Analysetechnik AG, Tübingen, Germany). FRET ratio was calculated as $\mathrm{F}_{535} / \mathrm{F}_{480}$ from background-corrected traces and given as FRET/FRET $_{\text {baseline }}$ (relative FRET ratio). Oligochrome control and fluorescence intensity acquisition was performed simultaneous to voltage clamp recordings using an EPC-10 USB patch clamp amplifier in combination with PatchMaster software (HEKA Electronics, Lambrecht, Germany).

Data analysis. Analysis and statistical testing of electrophysiological and imaging data were performed with IGOR Pro (WaveMetrics, Lake Oswego, OR, USA). Data are shown as mean \pm s.e.m. with numbers in the summary graphs indicating the number of experiments (individual cells or patches). Unless indicated otherwise, the two-tailed Dunnett's test was used for statistical testing. Asterisks denote the significance level, where ${ }^{* *}$ indicates $P<0.001{ }^{* *}$ indicates $P<0.01$, and * indicates $P<0.05$.

\section{References}

1. Brose, N., Betz, A. \& Wegmeyer, H. Divergent and convergent signaling by the diacylglycerol second messenger pathway in mammals. Curr. Opin. Neurobiol. 14, 328-340 (2004).

2. Kadamur, G. \& Ross, E. M. Mammalian phospholipase C. Annu. Rev. Physiol. 75, 127-154 (2013).

3. Oancea, E. \& Meyer, T. Protein kinase C as a molecular machine for decoding calcium and diacylglycerol signals. Cell 95, 307-318 (1998).

4. Carrasco, S. \& Merida, I. Diacylglycerol, when simplicity becomes complex. Trends Biochem. Sci. 32, 27-36 (2007).

5. Hurley, J. H. \& Misra, S. Signaling and subcellular targeting by membranebinding domains. Annu. Rev. Biophys. Biomol. Struct. 29, 49-79 (2000). 
6. Dietrich, A., Kalwa, H., Rost, B. R. \& Gudermann, T. The diacylgylcerolsensitive TRPC3/6/7 subfamily of cation channels: functional characterization and physiological relevance. Pflugers. Arch. 451, 72-80 (2005).

7. Enyedi, P. \& Czirjak, G. Molecular background of leak $\mathrm{K}^{+}$currents: two-pore domain potassium channels. Physiol. Rev. 90, 559-605 (2010).

8. Brickley, S. G. et al. TASK-3 two-pore domain potassium channels enable sustained high-frequency firing in cerebellar granule neurons. J. Neurosci. 27, 9329-9340 (2007).

9. Meuth, S. G. et al. Contribution of TWIK-related acid-sensitive $\mathrm{K}^{+}$channel 1 (TASK1) and TASK3 channels to the control of activity modes in thalamocortical neurons. J. Neurosci. 23, 6460-6469 (2003).

10. Perrier, J. F., Alaburda, A. \& Hounsgaard, J. 5-HT1A receptors increase excitability of spinal motoneurons by inhibiting a TASK-1-like $\mathrm{K}^{+}$current in the adult turtle. J. Physiol. 548, 485-492 (2003).

11. Duprat, F. et al. TASK, a human background $\mathrm{K}^{+}$channel to sense external $\mathrm{pH}$ variations near physiological pH. EMBO J. 16, 5464-5471 (1997).

12. Chemin, J. et al. Mechanisms underlying excitatory effects of group I metabotropic glutamate receptors via inhibition of $2 \mathrm{P}$ domain $\mathrm{K}^{+}$channels. EMBO J. 22, 5403-5411 (2003).

13. Mathie, A. Neuronal two-pore-domain potassium channels and their regulation by G protein-coupled receptors. J. Physiol. 578, 377-385 (2007).

14. Talley, E. M. \& Bayliss, D. A. Modulation of TASK-1 (Kcnk3) and TASK-3 (Kcnk9) potassium channels: volatile anesthetics and neurotransmitters share a molecular site of action. J. Biol. Chem. 277, 17733-17742 (2002).

15. Talley, E. M., Lei, Q., Sirois, J. E. \& Bayliss, D. A. TASK-1, a two-pore domain $\mathrm{K}^{+}$channel, is modulated by multiple neurotransmitters in motoneurons. Neuron 25, 399-410 (2000).

16. Lazarenko, R. M. et al. Motoneuronal TASK channels contribute to immobilizing effects of inhalational general anesthetics. J. Neurosci. 30, 7691-7704 (2010)

17. Bandulik, S., Penton, D., Barhanin, J. \& Warth, R. TASK1 and TASK3 potassium channels: determinants of aldosterone secretion and adrenocortical zonation. Horm. Metab. Res. 42, 450-457 (2010).

18. Chen, X. et al. Inhibition of a background potassium channel by Gq protein $\alpha$ subunits. Proc. Natl Acad. Sci. USA 103, 3422-3427 (2006).

19. Schiekel, J. et al. The inhibition of the potassium channel TASK-1 in rat cardiac muscle by endothelin-1 is mediated by phospholipase C. Cardiovasc. Res. 97, 97-105 (2013)

20. Horowitz, L. F. et al. Phospholipase $\mathrm{C}$ in living cells: activation, inhibition, $\mathrm{Ca}^{2+}$ requirement, and regulation of M current. J. Gen. Physiol. 126, 243-262 (2005).

21. Boyd, D. F., Millar, J. A., Watkins, C. S. \& Mathie, A. The role of $\mathrm{Ca}^{2+}$ stores in the muscarinic inhibition of the $\mathrm{K}+$ current $\mathrm{IK}(\mathrm{SO})$ in neonatal rat cerebellar granule cells. J. Physiol. 529(Pt 2): 321-331 (2000).

22. Czirjak, G., Petheo, G. L., Spat, A. \& Enyedi, P. Inhibition of TASK-1 potassium channel by phospholipase C. Am. J. Physiol. Cell Physiol. 281, C700-C708 (2001).

23. Stauffer, T. P., Ahn, S. \& Meyer, T. Receptor-induced transient reduction in plasma membrane PtdIns $(4,5) \mathrm{P}_{2}$ concentration monitored in living cells. Curr. Biol. 8, 343-346 (1998).

24. Varnai, P. \& Balla, T. Visualization of phosphoinositides that bind pleckstrin homology domains: calcium- and agonist-induced dynamic changes and relationship to Myo-[3H]inositol-labeled phosphoinositide pools. J. Cell Biol. 143, 501-510 (1998).

25. Oancea, E., Teruel, M. N., Quest, A. F. \& Meyer, T. Green fluorescent protein (GFP)-tagged cysteine-rich domains from protein kinase $\mathrm{C}$ as fluorescent indicators for diacylglycerol signaling in living cells. J. Cell Biol. 140, 485-498 (1998).

26. Venkatakrishnan, G. \& Exton, J. H. Identification of determinants in the $\alpha$-subunit of $\mathrm{G}_{\mathrm{q}}$ required for phospholipase $\mathrm{C}$ activation. J. Biol. Chem. 271, 5066-5072 (1996).

27. Zhang, X. et al. Direct inhibition of the cold-activated TRPM8 ion channel by $\mathrm{G} \alpha_{\mathrm{q}}$. Nat. Cell Biol. 14, 851-858 (2012).

28. Lopes, C. M. B. et al. $\mathrm{PIP}_{2}$ hydrolysis underlies agonist-induced inhibition and regulates voltage gating of two-pore domain $\mathrm{K}^{+}$channels. J. Physiol. 564, 117-129 (2005).

29. Lindner, M., Leitner, M. G., Halaszovich, C. R., Hammond, G. R. \& Oliver, D. Probing the regulation of TASK potassium channels by $\mathrm{PI}(4,5) \mathrm{P}_{2}$ with switchable phosphoinositide phosphatases. J. Physiol. 589, 3149-3162 (2011).

30. Hammond, G. R. et al. PI4P and PI $(4,5) \mathrm{P}_{2}$ are essential but independent lipid determinants of membrane identity. Science 337, 727-730 (2012).

31. Suh, B.-C., Inoue, T., Meyer, T. \& Hille, B. Rapid chemically induced changes of PtdIns(4,5) $\mathrm{P}_{2}$ gate KCNQ ion channels. Science 314, 1454-1457 (2006).

32. Bae, Y. S. et al. Identification of a compound that directly stimulates phospholipase C activity. Mol. Pharmacol. 63, 1043-1050 (2003)

33. Hodgkin, M. N. et al. Diacylglycerols and phosphatidates: which molecular species are intracellular messengers? Trends Biochem. Sci. 23, 200-204 (1998).

34. Maingret, F., Patel, A. J., Lazdunski, M. \& Honore, E. The endocannabinoid anandamide is a direct and selective blocker of the background $\mathrm{K}^{+}$channel TASK-1. EMBO J. 20, 47-54 (2001).
35. Reisenberg, M., Singh, P. K., Williams, G. \& Doherty, P. The diacylglycerol lipases: structure, regulation and roles in and beyond endocannabinoid signalling. Philos. Trans. R. Soc. Lond. B. Biol. Sci. 367, 3264-3275 (2012).

36. Aller, M. I. et al. Modifying the subunit composition of TASK channels alters the modulation of a leak conductance in cerebellar granule neurons. J. Neurosci. 25, 11455-11467 (2005).

37. Kang, D., Han, J., Talley, E. M., Bayliss, D. A. \& Kim, D. Functional expression of TASK-1/TASK-3 heteromers in cerebellar granule cells. J. Physiol. 554, 64-77 (2004)

38. Millar, J. A. et al. A functional role for the two-pore domain potassium channel TASK-1 in cerebellar granule neurons. Proc. Natl Acad. Sci. USA 97, 3614-3618 (2000).

39. Watkins, C. S. \& Mathie, A. A non-inactivating $\mathrm{K}^{+}$current sensitive to muscarinic receptor activation in rat cultured cerebellar granule neurons. J. Physiol. 491, 401-412 (1996).

40. Han, J., Truell, J., Gnatenco, C. \& Kim, D. Characterization of four types of background potassium channels in rat cerebellar granule neurons. J. Physiol. 542, 431-444 (2002)

41. Veale, E. L. et al. G $\alpha_{\mathrm{q}}$-mediated regulation of TASK3 two-pore domain potassium channels: the role of protein kinase C. Mol. Pharmacol. 71, 1666-1675 (2007).

42. Veale, E. L., Buswell, R., Clarke, C. E. \& Mathie, A. Identification of a region in the TASK3 two pore domain potassium channel that is critical for its blockade by methanandamide. Br. J. Pharmacol. 152, 778-786 (2007).

43. Bautista, D. M. et al. Pungent agents from Szechuan peppers excite sensory neurons by inhibiting two-pore potassium channels. Nat. Neurosci. 11, 772-779 (2008).

44. Hofmann, T. et al. Direct activation of human TRPC6 and TRPC3 channels by diacylglycerol. Nature 397, 259-263 (1999).

45. Chemin, J. et al. A phospholipid sensor controls mechanogating of the $\mathrm{K}^{+}$ channel TREK-1. EMBO J. 24, 44-53 (2005).

46. Honore, E., Maingret, F., Lazdunski, M. \& Patel, A. J. An intracellular proton sensor commands lipid- and mechano-gating of the $\mathrm{K}^{+}$channel TREK-1. EMBO J. 21, 2968-2976 (2002).

47. Sandoz, G., Bell, S. C. \& Isacoff, E. Y. Optical probing of a dynamic membrane interaction that regulates the TREK1 channel. Proc. Natl Acad. Sci. USA 108, 2605-2610 (2011).

48. Ashmole, I. et al. The response of the tandem pore potassium channel TASK-3 $(\mathrm{K}(2 \mathrm{P}) 9.1)$ to voltage: gating at the cytoplasmic mouth. J. Physiol. 587, 4769-4783 (2009).

49. Shulga, Y. V., Topham, M. K. \& Epand, R. M. Regulation and functions of diacylglycerol kinases. Chem. Rev. 111, 6186-6208 (2011).

50. Leach, N. T. et al. Disruption of diacylglycerol kinase delta (DGKD) associated with seizures in humans and mice. Am. J. Hum. Genet. 80, $792-799$ (2007).

51. Kakefuda, K. et al. Diacylglycerol kinase $\beta$ knockout mice exhibit lithiumsensitive behavioral abnormalities. PLoS ONE 5, e13447 (2010).

52. Rodriguez de Turco, E. B. et al. Diacylglycerol kinase epsilon regulates seizure susceptibility and long-term potentiation through arachidonoyl- inositol lipid signaling. Proc. Natl Acad. Sci. USA 98, 4740-4745 (2001).

53. Bodmann, E. L. et al. Dynamics of $\mathrm{G} \alpha_{\mathrm{q}}$-protein-p63RhoGEF interaction and its regulation by RGS2. Biochem. J. 458, 131-140 (2014).

54. Oliver, D. et al. Functional conversion between A-type and delayed rectifier $\mathrm{K}^{+}$ channels by membrane lipids. Science 304, 265-270 (2004).

55. Halaszovich, C. R., Schreiber, D. N. \& Oliver, D. Ci-VSP is a depolarizationactivated phosphatidylinositol-4,5-bisphosphate and phosphatidylinositol3,4,5-trisphosphate 5'-phosphatase. J. Biol. Chem. 284, 2106-2113 (2009).

\section{Acknowledgements}

We thank Drs J. Daut, J. Oberwinkler and T. Budde for helpful discussions and critica reading of the manuscript, and $\mathrm{M}$. Bertoune for help with establishing granule neuron cultures. This work was supported by grants from the Deutsche Forschungsgemeinschaft to D.O. (FOR 1086 OL 240/3-1 and SFB 593 TP12)

\section{Author contributions}

D.O. and B.U.W. conceived the study; B.U.W., M.L., L.G., A.A., Y.K. and M.G.L. performed experiments and analysed data; D.O. analysed data; M.B. developed the FRET assay; D.O. and B.U.W. wrote the manuscript.

\section{Additional information}

Competing financial interests: The authors declare no competing financial interests.

Reprints and permission information is available online at http://npg.nature.com/ reprintsandpermissions/

How to cite this article: Wilke, B. U. et al. Diacylglycerol mediates regulation of TASK potassium channels by Gq-coupled receptors. Nat. Commun. 5:5540 doi: $10.1038 /$ ncomms6540 (2014). 